\title{
Correspondence
}

\section{The Beneficial Effects of Statin Therapy May Not Apply to All Forms of Crescentic Glomerulonephritis}

To the Editor-in-Chief:

We read with interest the manuscript by Fujita et al ${ }^{1}$ describing the effect of statin treatment on experimental anti-glomerular basement membrane glomerulonephritis (anti-GBM GN) in WKY rats. In their elegant study, they showed that atorvastatin (20 mg/kg/day p.o.) given three days pre-induction of $\mathrm{GN}$ and for up to 10 days markedly reduced the degree of renal injury. They attributed this to reduction in pro-inflammatory $\mathrm{M} 1$ macrophages and increase in anti-inflammatory M2 macrophages. The authors propose a therapeutic application for statins in severe glomerulonephritis.

Their results contrast with the effect of simvastatin in rodent experimental autoimmune vasculitis (EAV), an analogous model of crescentic GN induced by immunization with myeloperoxidase (MPO). ${ }^{2-3}$ We gave simvastatin (Rosemont Pharmaceuticals, Leeds, UK) at a similar dose of $25 \mathrm{mg} / \mathrm{kg}$ body weight/day p.o. from five days pre-immunization and continued for 56 days. Despite having a potent in vitro effect on anti-MPO antibody-induced neutrophil activation, we found that simvastatin did not reduce hematuria, albuminuria, or the degree of crescentic GN. ${ }^{4}$ Taking this in conjunction with a recent case report of vasculitis apparently caused by a statin, ${ }^{5}$ we propose that the effect of statin therapy may not apply to all forms of crescentic glomerulonephritis and advise caution in extrapolating to human disease.

Bahjat Al-Ani

Hamad Al Nuaimi

Caroline Savage

University of Birmingham

Birmingham, UK

Mark A. Little

UCL Centre for Nephrology Royal Free Hospital

London, UK

\section{Author's Reply:}

We thank Al-Ani et al for their interest in our recent publication, ${ }^{1}$ and we are impressed by the extensive and important work the group has achieved contributing to the field of crescentic glomerulonephritis (GN) research. As described in their letter, their research model of human myeloperoxidase (MPO)-immunized rodent antineutrophil cytoplasmic antibody (ANCA)-associated systemic vasculitis is quite interesting and considered one that best resembles human ANCA-associated systemic vasculitides. Their recent study showed that although simvastatin reduced anti-MPO antibody-induced neutrophil degranulation and VWF release from normal human umbilical vein endothelial cells co-cultured with ANCAstimulated neutrophils, administration of simvastatin did not ameliorate hematuria, albuminuria, or the degree of glomerular injury in rats. We are not surprised by these negative results in vivo, given that statin sometimes enhances humoral immune responses and may sometimes be associated with drug-induced autoimmune reactions, including systemic lupus erythematosus as well as ANCA-associated vasculitis. ${ }^{6}$ We speculate that these enhanced immune responses may have occurred via activated Th2-promoting effects, ${ }^{7}$ causing augmentation of humoral immune responses in autoimmune reactions. Evidence suggest that statins are also sometimes capable of inducing proinflammatory responses and acting in a disease-specific manner. ${ }^{8}$ In fact, statin therapy does not seem to benefit giant cell arteritis, ${ }^{9}$ one of the systemic vasculitides.

In contrast, recent observations suggest that statin treatment may have renoprotective effects. ${ }^{10-12}$ The benefits of statins include lipid lowering as well as pleiotrophic effects. The pleiotrophic effects may derive from inhibition of the mevalonate pathway and the synthesis of the isoprenoids (farnesyl pyrophosphate and geranylgeranyl pyrophosphate) involved in cellular signaling, post-translational modification of proteins, and cellular function. ${ }^{13}$ The pleiotrophic effects of statins, such as endothelial protection and antiinflammatory, oxidative, and thrombotic events, may eventually provide renal benefits. ${ }^{10-13}$

Our results revealed that atorvastatin can suppress glomerular inflammation via inhibition of macrophage development, reduction of pro-inflammatory M1 macrophages, and augmentation of anti-inflammatory M2 macrophages. ${ }^{1}$ In renal glomerular diseases, activated macrophages contribute to the progression of glomerular inflammation. ${ }^{14}$ Dendritic cells and T cells are also involved in this process. ${ }^{15}$ 
Statins directly affect multiple cell populations relevant to immune response such as dendritic cells, T cells, regulatory $\mathrm{T}$ cells, macrophages, B cells, and endothelial cells, thereby promoting an anti-inflammatory phenotype opposed to pro-inflammatory phenotype. 12,13,16,17 Based on these findings, it is highly probable that the protective effects of statins, together with their cholesterol-lowering effects, can help treat glomerular diseases. In support of this concept, accumulating evidence has been published using experimental models of renal diseases, including our recent report. ${ }^{1,10-12,18}$ However, renoprotective effects of statins in human renal diseases are not well established. ${ }^{10-12,19} \mathrm{Ad}-$ ditional prospective, controlled, randomized trials are needed to assess the efficacy of statins in that respect.

We agree that the effect of statin therapy may not apply to all forms of crescentic GN and advise caution in extrapolating to human disease. It is very important that large trial studies be performed to clarify whether statin therapy is truly effective in human severe GN, including crescentic GN and ANCA-associated systemic vasculitis.

Nippon Medical School

Tokyo, Japan

\section{References}

1. Fujita E, Shimizu A, Masuda $Y$, Kuwahara N, Arai T, Nagasaka S, Aki K, Mii A, Natori $Y$, lino $Y$, Katayama $Y$, Fukuda $Y$ : Statin attenuates experimental anti-glomerular basement membrane glomerulonephritis together with the augmentation of alternatively activated macrophages. Am J Pathol 2010, 177:1143-1154

2. Little MA, Smyth L, Salama AD, Mukherjee S, Smith J, Haskard D, Nourshargh S, Cook HT, Pusey CD: Experimental autoimmune vasculitis: an animal model of anti-neutrophil cytoplasmic autoantibody-associated systemic vasculitis. Am J Pathol 2009, 174:12121220

3. Little MA, Smyth CL, Yadav R, Ambrose L, Cook HT, Nourshargh S, Pusey CD: Antineutrophil cytoplasm antibodies directed against my- eloperoxidase augment leukocyte-microvascular interactions in vivo. Blood 2005, 106:2050-2058

4. Al-Ani B, Al Nuaimi B, Bevins A, Plant T, Savage C, Little M: Inhibition of ANCA-Induced Neutrophil Stimulation by Simvastatin. Manchester, Renal Association, 2010, pp 425

5. Sen D, Rosenstein ED, Kramer N: ANCA-positive vasculitis associated with simvastatin/ezetimibe: expanding the spectrum of statininduced autoimmunity? Int J Rheum Dis 2010, 13:e29-e31

6. Noël B: Lupus erythematosus and other autoimmune diseases related to statin therapy: a systematic review. J Eur Acad Dermatol Venereol 2007, 21:17-24

7. Dunn SE, Youssef S, Goldstein MJ, Prod'homme T, Weber MS, Zamvil SS, Steinman L: Isoprenoids determine Th1/Th2 fate in pathogenic T cells, providing a mechanism of modulation of autoimmunity by atorvastatin. J Exp Med 2006, 203:401-412

8. Zeiser R, Maas K, Youssef S, Dürr C, Steinman L, Negrin RS: Regulation of different inflammatory diseases by impacting the mevalonate pathway. Immunology 2009, 127:18-25

9. Narváez J, Bernad B, Nolla JM, Valverde J: Statin therapy does not seem to benefit giant cell arteritis. Semin Arthritis Rheum 2007, 36: 322-327

10. Mason JC: The statins-therapeutic diversity in renal disease? Curr Opin Nephrol Hypertens 2005, 14:17-24

11. D'Amico G: Statins and renal diseases: from primary prevention to renal replacement therapy. J Am Soc Nephrol 2006, 17(4 Suppl 2): S148-S152

12. Campese VM, Park J: HMG-CoA reductase inhibitors and the kidney. Kidney Int 2007, 71:1215-1222

13. Greenwood J, Steinman L, Zamvil SS: Statin therapy and autoimmune disease: from protein prenylation to immunomodulation. Nat Rev Immunol 2006, 6:358-370

14. Matsumoto K, Fukuda N, Abe M, Fujita T: Dendritic cells and macrophages in kidney disease. Clin Exp Nephrol 2010, 14:1-11

15. Kurts C, Heymann F, Lukacs-Kornek V, Boor P, Floege J: Role of T cells and dendritic cells in glomerular immunopathology. Semin Immunopathol 2007, 29:317-335

16. Zhang X, Markovic-Plese S: Statins' immunomodulatory potential against Th17 cell-mediated autoimmune response. Immunol Res 2008, 41:165-174

17. Kim YC, Kim KK, Shevach EM: Simvastatin induces Foxp3+ T regulatory cells by modulation of transforming growth factor-beta signal transduction. Immunology 2010, 130:484-493

18. Eller P, Eller K, Wolf AM, Reinstadler SJ, Tagwerker A, Patsch JR, Mayer G, Rosenkranz AR: Atorvastatin attenuates murine anti-glomerular basement membrane glomerulonephritis. Kidney Int 2010, 77: 428-435

19. Sandhu S, Wiebe N, Fried LF, Tonelli M: Statins for improving renal outcomes: a meta-analysis. J Am Soc Nephrol 2006, 17:20062016 